\section{rev Psi}

Revista de Psicología (UNLP)

https://revistas.unlp.edu.ar/revpsi

\title{
El bienestar emocional en madres de hijos con diagnóstico de TEA
}

\author{
María José Bagnato ${ }^{1}$ \\ Correspondencia \\ majose@psico.edu.uy \\ Filiaciones institucionales \\ ${ }^{1}$ Facultad de Psicología, Universidad de la \\ República (Uruguay)
}

Eugenia Barboza ${ }^{1}$

\section{Resumen}

Presentamos resultados de un estudio exploratorio sobre bienestar emocional de madres de un centro formado por una organización referente en Trastornos del Espectro Autista (TEA) del departamento de San José, Uruguay. Proponemos un avance de resultados y análisis que corresponde al primer objetivo del estudio mayor "Incidencia del cuidado en la calidad de vida de madres de hijos/as con discapacidades severas de origen psíquico", desarrollado por el Programa Discapacidad y Calidad de Vida (Facultad de Psicología, Universidad de la República). Se plantean los objetivos: (a) describir el bienestar emocional de las madres participantes del estudio; y (b) describir qué aspectos del bienestar emocional se relaciona con el hecho de tener un/a hijo/a con diagnóstico de TEA. Se propone el análisis de los resultados en función de la dimensión Bienestar Emocional del concepto calidad de vida familiar propuesto por Ann Turnbull.

\section{Palabras clave}

bienestar emocional $\mid$ madres $\mid$ TEA $\mid$ discapacidad

\section{Cómo citar}

Bagnato, M.J. y Barboza, E. (2019). El

bienestar emocional en madres de hijos con diagnóstico de TEA. Revista de Psicología, 18(1), 46-60. doi: $10.24215 / 2422572 \mathrm{Xe} 027$
Recibido

3 sep. 2018

Aceptado

4 dic. 2018

Publicado

13 may. 2019

Editor

Nicolás Alessandroni | Facultad de Psicología, Universidad Autónoma de Madrid (España)
ISSN

2422-572X

Licencia

(c) Copyright: Bagnato, M. J. y Barboza, E. El trabajo se distribuye bajo licencia Cultura Libre CC-BY 4.0

Entidad editora

RevPsi es una publicación de la

Facultad de Psicología (Universidad Nacional de La Plata, Argentina)

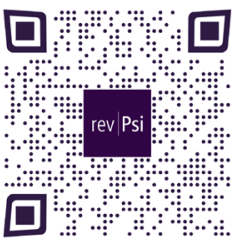

ACCESO ABIERTO DIAMANTE 


\section{Bem-estar emocional em mães de crianças diagnosticadas com TEA}

\section{Resumo}

Apresentamos resultados de um estudo exploratório sobre o bem-estar emocional de mães de um centro formado por uma organização de referência em Distúrbios do Espectro Autista (ASD) do departamento de San José, no Uruguai. Propomos um avanço de resultados e análise que corresponda ao primeiro objetivo do estudo maior "Incidência de cuidado na qualidade de vida de mães de crianças com graves deficiências de origem psíquica", desenvolvido pelo Programa de Incapacidade e Qualidade de Vida (Faculdade de Enfermagem). Psicologia, Universidade da República. Os objetivos são: (a) descrever o bem-estar emocional das mães participantes do estudo; e (b) descrever quais aspectos do bem-estar emocional estão relacionados a ter um filho diagnosticado com TEA. A análise dos resultados é proposta de acordo com a dimensão Bem Estar Emocional do conceito de qualidade familiar proposta por Ann Turnbull.

\section{Palavras-chave}

bem-estar emocional | mães | TEA | incapacidade

\section{Emotional well-being in mothers of children diagnosed with ASD}

\section{Abstract}

We present results of an exploratory study on the emotional well-being of mothers of a center created by a leading organisation working in Autism Spectrum Disorders in the department of San José, Uruguay. We advance results and analyses that corresponds to the first objective of the larger study "Incidence of care in the quality of life of mothers of children with severe disabilities of psychic origin", developed by the Disability and Life Quality Program (Faculty of Psychology, University of the Republic). Our objectives are: (a) to describe the emotional well-being of the mothers participating in the study; and (B) to describe what aspects of emotional well-being are related to having a child diagnosed with ASD. The analysis of the results was done according to the Emotional Well-Being dimension of the concept of family quality of life proposed by Ann Turnbull.

\section{Keywords}

emotional well-being $\mid$ mothers | ASD | disability 


\section{Aspectos destacados del trabajo}

- Se explora el bienestar emocional en madres de niños con TEA, un tópico poco investigado.

- El análisis considera la dimensión Bienestar Emocional del concepto calidad de vida familiar de Turnbull.

- Se incluyen fragmentos de testimonios que son analizados cualitativamente.

- Destaca el lugar que dan las madres a agrupaciones de familiares para afrontar situaciones adversas.

\section{Introducción y marco teórico}

El bienestar emocional es una de las dimensiones que componen el constructo calidad de vida (CDV), ya sea de la persona individualmente considerada o cuando se analiza la calidad de vida familiar. Si bien hay escasa investigación sobre el impacto en la CDV de los cuidadores familiares de personas con discapacidades severas, la existente confirma que éstos enfrentan una serie de dificultades debido a la problemática compleja de sus hijos (Aubeeluck, Buchanan y Stupple, 2012). En la mayoría de las situaciones, el contar con un diagnóstico de Trastorno del Espectro Autista (TEA) es un componente importante para determinar que se está ante una situación de discapacidad. Si bien el diagnóstico, de por sí, no se conceptualiza como discapacidad en el paradigma actual (CIF-OMS, 2001), la presencia de limitaciones significativas en la conducta adaptativa que puede coexistir o no con limitaciones del funcionamiento intelectual e impactar en el relacionamiento, conduce frecuentemente a situaciones de restricciones en la participación. En definitiva, para la inclusión social.

Es habitual observar las barreras sociales que ocurren ante la incomprensión de las conductas que presentan las personas diagnosticadas con TEA. La situación de discapacidad es relativa a las posibilidades de inclusión: cuando existen déficits significativos en la conducta adaptativa, los niños/as intensifican sus demandas hacia las familias y, en particular, a las madres, incidiendo en su bienestar (Merino, Martínez, Cuesta, García y Pérez de la Varga, 2012). Brasfield (2008), basada en investigaciones previas (Beck, Hastings, Daley y Stevenson, 2004; Kobe y Hammer, 1994; Lessenberry y Rehfeldt, 2004; Martin y Baker, 2001; Withers y Bennett, 2003), plantea que el bienestar emocional de la madre afecta al del hijo, el desarrollo físico y el progreso en el tratamiento. Asimismo, por parte de los padres aparecen afectaciones en lo emocional, quienes suelen atravesar un proceso que implica hipersensibilidad, 
labilidad, incertidumbre respecto a la situación familiar, sentimiento de culpabilidad y/o enojo, lo cual influye directamente en el trato con el hijo.

Este estudio se dirige a las madres, dados los antecedentes que plantean que son las madres (abuelas, tías, hermanas) de los niños quienes dedican la mayor cantidad de horas al cuidado (Martínez y Bilbao, 2008), pero ello no significa que se considere que los padres estén ausentes. Detrás de estas comprobaciones, existe la sombra de la inequidad de género, con relación a la concepción acerca del rol "tradicional" que asumen las madres respecto del cuidado del grupo familiar (Gómez, 2008).

La obtención de un diagnóstico de TEA, así como de otra discapacidad severa en la etapa infantil, provoca en la familia una situación de crisis. La crisis puede ser un estado provisorio, temporal, esperable frente a esta situación o puede sostenerse en el tiempo desencadenando diversas consecuencias desfavorables. Que se visualice un camino u otro, está íntimamente ligado a varios factores, entre ellos, fundamentalmente, la presencia o ausencia de apoyo social y familiar. La crisis a la que se ve enfrentada la familia puede derivar en "crecimiento" o generar un importante deterioro psicológico. Uno u otro destino, depende directamente de la singularidad de cada familia, sus características y los recursos a los que pueda acceder (Canal Bedia et al., 2006).

Datos generales sobre la población con discapacidad en Uruguay

En Uruguay, según los datos del último Censo Nacional (2011), considerando las edades entre 0 y 14 años, de 709.506 relevados, 25.907 niños se reportan con al menos una discapacidad leve, 9037 con una discapacidad moderada y 1786 con una discapacidad severa. Si tomamos en cuenta aquellos que presentan específicamente limitaciones para aprender y/o entender, el criterio utilizado para relevar la información fue para mayores de 6 años, lo que coincide con la edad escolar. En este caso, de un total de 446.007 niños, 54.331 reportan tener alguna limitación, 5.662 mucha limitación, mientras que 704 reportan no poder aprender. De acuerdo a datos presentados por el Banco de Previsión Social (BPS) mediante la Encuesta Longitudinal de Protección Social (2012-2013), del total de personas con al menos una limitación el 22.8 por ciento son de relacionamiento y/o aprendizaje y, de ese grupo de personas, el 24 por ciento son menores de 15. Sin embargo, no se cuenta con información fiable acerca del número de personas incluidas en el diagnóstico de TEA.

Viola, Garrido y Varela (2008) afirman que Uruguay no cuenta con estudios epidemiológicos nacionales en el área de Salud Mental infantil. Las autoras llevaron a cabo un estudio que recogió la percepción de los padres sobre la presencia o ausencia de síntomas comportamentales o emocionales de sus hijos entre 6 y 11 años. El estudio establece que el 22 por ciento de los niños/as uruguayos presentan problemas conductuales o emocionales de entidad, que entrarían en la categoría de 
diagnosticables. Si bien existe cierta información sobre población con discapacidad, no existen en el país datos confiables, específicos y representativos sobre la proporción de la población con discapacidad que es dependiente, ni sobre el tipo de dependencia que presentan (Bagnato, Da Cunha y Falero, 2011). Por lo dicho, son necesarios estudios que establezcan cuál es la prevalencia de discapacidad y dependencia, por lo que, en particular, aún es más difuso definir la prevalencia de la población con discapacidad infantil que vincula problemáticas mentales e intelectuales, independientemente del origen de la discapacidad (deficiencia). Se considera que contar con datos cualitativos, que describan las situaciones de las familias, puede dar cuenta de la necesidad de contar con mayores datos a nivel poblacional.

\section{Calidad de vida familiar}

El uso del concepto de calidad de vida ha crecido de manera significativa desde los 60 , pero es en los últimos quince años que se ha observado un importante crecimiento de investigaciones en diversos campos como la salud mental, la salud, la educación, la discapacidad, los servicios y el mundo laboral (Verdugo Alonso y Sabeh, 2002; Bagnato, 2009). Desde la psiquiatría y la psicología se realizan evaluaciones de DCV con el fin de medir los resultados de programas y de terapias para enfermos crónicos. También se han estudiado las repercusiones del grado de apoyo social, el funcionamiento individual y el nivel de autonomía en la calidad de vida (Gómez y Sabeh, 2001). Es importante comprender que el concepto se encuadra a partir de una forma de concebir al sujeto y su familia. Ésta ha ido cambiando a través del tiempo y responde al nuevo paradigma de la discapacidad presente en la actualidad: "los modelos mentales están fuertemente arraigados en hipótesis, generalizaciones y representaciones mentales que tenemos para comprender el mundo" (Schalock y Verdugo Alonso, 2007, p. 7). Éstos son los que conforman la cultura y visión de una organización y sirven de base para dirigir nuestra práctica profesional, formar en valores, desarrollar los servicios, evaluar los resultados y mejorar en calidad. Según Shalock y Verdugo Alonso (2007), en la discapacidad son varios los modelos que no han permitido los cambios históricamente. Ann Turnbull (2003) plantea en consonancia, que el puente que ha servido para pasar del viejo paradigma de la discapacidad a uno nuevo ha sido el valor que se le ha asignado a la investigación centrada en la familia y los servicios con los que cuenta.

El modelo o enfoque centrado en la familia es un modelo de intervención profesional, formado por principios, por valores y por prácticas, que otorga a la familia un papel fundamental en la planificación, la provisión y la evaluación de los servicios. Los objetivos se basan en el empoderamiento o capacitación familiar, y en la mejora de la calidad de vida personal y familiar (Peralta López y Arellano Torres, 2010). Este modelo, se caracteriza por tres factores principales: en primer lugar, se cambia la relación de poder entre profesionales y familias, los profesionales responden a las prioridades de la familia; en segundo lugar, abandona la orientación basada 
en la patología para pasar a una que se centre en las potencialidades y, en tercer lugar, toda la familia es considerada como la unidad de apoyo y no sólo el niño con discapacidad y su madre (Turnbull, 2003). Desde este modelo en el que se prioriza la capacitación funcional de la familia, el equipo del Centro Beach de Discapacidad de la Universidad de Kansas (Estados Unidos), propuso una conceptualización multidimensional de la calidad de vida familiar.

Cuando se diagnostica un TEA, se hace referencia a un conjunto de problemas vinculados al neurodesarrollo. Éstos presentan manifestaciones principalmente cognitivas y comportamentales, que producen grandes limitaciones en la autonomía personal y son una causa importante de estrés en la familia (Cabanyes-Truffino $y$ García-Villamisar, 2004). Es considerado un trastorno de etiología múltiple, más allá de que no hay una respuesta concluyente acerca de la naturaleza y las causas que lo originan y, estudios recientes, han demostrado una responsabilidad genética en la etiología (Pozo, 2010). En la actualidad, no se disponen marcadores biológicos de los TEA, por lo que el diagnóstico depende, fundamentalmente, de sus manifestaciones clínicas. De este modo, se definen por la presencia de alteraciones de tipo cualitativo en la interacción social y la comunicación y por la restricción del repertorio de intereses, de conductas y de actividades (Cabanyes-Truffino y García-Villamisar, 2004).

El presente estudio pretende aportar resultados con relación a cómo viven las madres cuidadoras el proceso de crianza de sus hijos con diagnóstico de TEA, en cuanto a cómo impacta en su calidad de vida. Si bien todas las dimensiones de la calidad de vida se encuentran interrelacionadas, en particular, hemos seleccionado la dimensión Bienestar Emocional, ya que entendemos que la percepción de este bienestar es un importante resonador de las demás áreas o dimensiones.

\section{Objetivos}

Para analizar el bienestar emocional nos propusimos los siguientes objetivos específicos:

- describir el bienestar emocional de las madres participantes del estudio;

- describir qué aspectos del bienestar emocional se relaciona con el hecho de tener un/a hijo/a o más con diagnóstico de TEA.

\section{Metodología}

Se trata de un estudio exploratorio, preliminar del estudio mayor: "Incidencia del cuidado en la calidad de vida de madres de hijos/as con discapacidades severas de origen psíquico", actualmente en ejecución. La metodología utilizada para este trabajo 
se enmarca en el paradigma cualitativo, ya que constituye una "visión de mundo", una manera sistémica, estructurada de percibir, de aprehender, de comprender, de explicar y de argumentar la naturaleza humana y las características de los objetos que componen el universo, objeto de interés de la ciencia (Rodríguez, 2011).

La población a estudiar se conforma por las madres de hijos con un diagnóstico de Trastorno de Espectro Autista que asisten al Centro Florecer en San José de Mayo (Uruguay). Se seleccionó esta población por estar ubicada en el interior del país teniendo en cuenta que es la primera Asociación de padres de personas con Autismo formada en Uruguay y es la encargada de que el proyecto se multiplique en los distintos departamentos del país.

\section{Selección de la muestra}

El Centro Florecer está formado por 40 familias. Por ello, se decidió hacer una selección para llegar a una muestra significativa, definida ésta como un subconjunto limitado de miembros de una población que se investiga, con el fin de luego extender a toda la población las conclusiones que resulten del análisis de las informaciones relativas al subconjunto (Marradi, 2007).

Para la selección se tuvieron en cuenta algunos criterios de inclusión: uno de ellos fue no integrar a madres que estuvieran cursando grandes momentos de angustia, como también se evaluó seleccionar madres que no estuvieran en tratamiento psiquiátrico, es decir, tomando medicación. Se hizo considerando una heterogeneidad en las edades y en las experiencias de vida, así como en las situaciones económicas que presentaban cada una de ellas. Sobre la base de estos criterios, se seleccionaron once madres de la institución, a las cuáles se les realizó la invitación de participar de forma personal, explicando en qué consistía la investigación mayor, mencionando que cuenta con el aval de ética necesario, definiendo los objetivos que se plantean, desarrollando la importancia que tienen en la investigación y que se conservaría la confidencialidad de los datos. También se mencionó que las técnicas aplicadas se utilizarían para la presente investigación, mencionando todas las características y sus objetivos, firmando cada una de ellas un consentimiento informado.

El subconjunto fue formado, en un principio, por once madres que cumplían con los criterios de inclusión en el grupo y accedieron a participar en su totalidad representando el 22.5 por ciento de la población total del centro. El rango de edad va desde los 30 a los 55 años y la de sus hijos de 3 a los 30 años.

\section{Técnica empleada}

La técnica seleccionada para la obtención de datos, enmarcada en el enfoque cualitativo fue el grupo focal (focus group), es decir, un tipo de entrevista grupal. Su dinámica se basa en organizar un grupo de personas para que discutan de un 
tema determinado objeto de la investigación, registrando la información obtenida producto de la interacción que refleja las experiencias grupales y las distintas perspectivas, para luego clasificarla y analizarla con el fin de elaborar conclusiones y sugerencias. La decisión de seleccionar esta técnica se vincula al hecho de la riqueza que brinda la situación social de las personas hablando desde un lugar más distendido, donde no se da sólo una opinión individual aislada, sino que son respuesta a la situación de interacción, de debate, compartiendo esa instancia con otras madres en la misma situación, donde sus apreciaciones se van entrelazando con las de otros participantes. Además, permite ver desde un escenario todos los elementos que se ponen en juego en una discusión grupal: "actitudes, emociones, creencias, opiniones, experiencias, evaluaciones, reacciones, consensos y disensos. Estos elementos serán articulados luego -a través del análisis - en estructuras de sentido" (Marradi, 2007).

Las preguntas realizadas fueron presentadas de forma abierta, buscando promover la participación y teniendo en cuenta los objetivos del estudio. A su vez, se elaboró un guión que permitiera el despliegue de la opinión de las madres en torno a ejes principales en relación al cuidado de sus hijos, a partir del cual se identificará el bienestar emocional como dimensión de análisis. Las preguntas del guión fueron:

- ¿Cuáles son los principales problemas que identifican con sus hijos?

- ¿Cuáles son las estrategias de afrontamiento (cómo hacen para resolver los problemas)?

- ¿Cuáles son las necesidades que identifican?

- ¿Cuentan con ayuda de acompañante o asistente personal? En el caso de que sí ¿les ha sido de ayuda?

El grupo focal se realizó en una sesión que fue grabada y desgravada en su totalidad. Así, las madres expresaron abiertamente sus principales dificultades, así como las necesidades que surgen a la hora de tener un hijo con TEA y abrieron el debate acerca de variadas temáticas contempladas en los objetivos de la investigación. Con respecto a las áreas de la calidad de vida familiar, se obtuvo información sustantiva que da visibilidad al impacto, la experiencia diaria y los desafíos presentes.

Para analizar la información, se realizó un cuadro de doble entrada, cuyas filas estaban representadas por cada participante y las columnas por las cinco áreas de la calidad de vida familiar. Se fue ubicando lo que manifestaba cada madre con respecto a cada una de esas áreas en el cuadrante correspondiente. A los efectos del presente reporte, se tomará exclusivamente la dimensión de Bienestar Emocional.

El concepto calidad de vida familiar propone cinco dimensiones: el apoyo relacionado con la discapacidad, el bienestar físico/material, el ser padres, el bienestar emocional 
y las interacciones familiares (Verdugo Alonso, Rodríguez Aguilella y Sainz Modino, 2012). A su vez, dentro de bienestar emocional, se definen una serie de indicadores: "Mi familia cuenta con el apoyo necesario para aliviar el estrés"; "los miembros de mi familia tienen amigos u otras personas que les brindan su apoyo"; "disponen de algún tiempo para ellos"; "mi familia cuenta con ayuda externa para atender a las necesidades especiales de todos los miembros de la familia".

Para exponer los resultados se seleccionan, mediante transcripción de la opinión de las participantes, algunas frases que permiten clarificar el contenido de la vivencia cotidiana que es compartida por las madres del grupo. Es así como, a partir de los componentes de la definición señalada, se identifican los contenidos que surgen de las madres participantes y se relacionan con la dimensión objeto de análisis.

\section{Tiempo de cuidado y tiempo libre}

En primer lugar, no tener tiempo para sí, parece ser uno de los signos de agotamiento emocional y físico más importantes. Esto puede deberse a que al estar concentrados los cuidados principalmente en las madres, no tienen posibilidades de encontrar momentos de descanso. A su vez, por las propias características de su hijo, no resulta fácil que personas accedan a relevar el cuidado, inclusive en su familia: "la mayor era eso, el a veces no encontrar un tiempo para uno porque las 24 horas eran para la atención de Flor" (participante 1).

Muchas veces, los únicos momentos de tiempo "libre" o "vividos como libres" suelen ser ir a trabajar cuando esto es posible, ya que hay casos donde las madres no lo hacen para encargarse de su hijo o cuando tienen que salir por otras responsabilidades inevitables: "yo tuve una época en que él estaba horrible, e iba a la mutualista a sacar medicamentos y me sentía en el paraíso, "hay que esperar acá, no me importa" y me sentía re bien" (participante 4) o como afirma el participante 6, "yo descanso en mi trabajo, ¡y mirá que trabajo!”.

Las madres del Centro representan las cuidadoras principales de sus hijos en su casa. Como se mencionó anteriormente, el cuidado principal de una persona con discapacidad conlleva una gran exigencia de energía, de tiempo, de desgaste físico y emocional. Es por este motivo que, si no se cuenta con apoyos necesarios, puede llevar a un desgaste en la salud importante. El Sistema Nacional Integrado de Cuidados brinda el asistente personal para personas con altos grados de dependencia, lo que contribuye a brindar al cuidador principal un tiempo de descanso. Este sistema ha permitido que las madres tengan tiempo para ellas, siendo un apoyo necesario para que logren hacer cosas tan básicas como bañarse, dormir, o caminar con tranquilidad. El servicio es evaluado de forma positiva y necesaria: "la entrego y me voy, así sea a caminar" (participante 6).

Lo obtuve [...] en ese momento no estaba trabajando, estaba con médica, porque no podía trabajar, porque no dormía entonces iba a trabajar y no existía [...] y me salió, la verdad que son cuatro horas por día de lunes a viernes, vos lo utilizás 
como querés, la verdad que esas cuatro horas a mí me parecen hasta eternas, yo volvía antes...y la ayudaba a ella a hacer cosas, y me decía "no, estoy yo acá, vos andá tranquila... ahora ya no, me desaparezco" (participante 8).

Más allá de que el Sistema ha tenido efectos en compartir la carga del cuidado que históricamente se ha concentrado en las mujeres y se base en principios de equidad (perspectiva de género) y autonomía (favorecer y promover la autonomía de la persona en situación de dependencia), cuando se trata de familias con un integrante con diagnóstico TEA, siguen presentes las necesidades con respecto a contar con espacios donde dejar a sus hijos, que estén adaptados para atender a esta población adecuadamente por cantidades prolongadas de tiempo (vacaciones, fin de semana o, incluso, emergencias). Irse de vacaciones o los fines de semana que presentan cambios en la rutina habitual, pueden generar grandes desafíos para las madres y para la persona con TEA. De esta manera, éstas no pueden acceder a salir a pasear o irse de vacaciones para no exponerse a situaciones difíciles que les generen estrés tanto a ello como a sus hijos: "(...) te pasa algo, o te querés ir de vacaciones diez días, y yo sé que Fiorella disfruta cierta cantidad de días después yo me vengo porque Fiorella ya no aguanta más, pero yo me quedaría" (participante 1). Por este motivo, plantean la necesidad de contar con un lugar que permita que puedan tener momentos de disfrute del tiempo libre, valorado por ellas como fundamental y muy lejos de su alcance.

\section{Aislamiento}

Las características propias de su hijo/a y principalmente los problemas de conducta hacen que prefieran no salir a ciertos lugares, ver amigos o familiares, por no pasar momentos que pueden terminar siendo estresantes, como por ejemplo regular una crisis:

No voy a un lugar porque por ejemplo iba a comidas con mis amigos y me iba, "ay quedate, que no le pasa nada, un berrinche, un ataque", "no, esto termina en algo peor, no me importa yo me voy" (participante 6).

(...) Me cambió la vida cien por ciento, yo una persona que me encantaba salir, salir todo el tiempo, que tenía amistades por todos lados, tuve que dejar un trabajo, ahora conseguí otro más accesible porque tuve suerte, pero de menos horas, la voy llevando ahí, aunque me cuesta horrores ir a trabajar... (participante 7).

Estas experiencias son reafirmadas por otros participantes al decir "yo me aislé por completo" (participante 6) o "el trastorno te pasa a vos, como que trasciende y te volvés un poco autista" (participante 1). Estas son algunas de las frases que dan cuenta de la tendencia de las madres a dejar de participar de espacios con otros, espacios de socialización, por tanto de aislamiento. 


\section{Áreas problemáticas de la conducta adaptativa}

Así como las conductas no adaptativas de sus hijos tienen repercusiones en cómo las madres viven su tiempo libre y en los vínculos que establecen, también suelen ser un desafío constante en el día a día y representan en lo cotidiano grandes obstáculos que afectan su estado emocional, las oportunidades y determinadas conductas implican que éstas tengan que cambiar características propias de su funcionamiento para sobrellevar el día a día.

Lo manifiestan explícitamente diciendo: "te tenés que adaptar o te morís" (participante 4), "muchas cosas las regula ella" (participante 1) o "es un trastorno para toda la vida, no es un cáncer... fue el diagnóstico que le dieron a nuestro hijo y él va a ser así, o te acostumbrás o te morís" (participante 2).

Estas situaciones a las que se ven expuestas cotidianamente pueden llevar a que se desborden, ya que hay sentimientos de angustia y desmotivación, llegando a límites donde no se ven salidas posibles: "a veces te entra una cosa de, ay por dios, esto, te querés matar" (participante 4), "te avanza la depresión y te mata " (participante 2), "te querés morir, te querés matar y te querés ir al diablo, eso yo por lo menos lo pasé" (participante 3) o "antes creía que me iba a enloquecer" (participante 6).

Estas expresiones corresponden a las madres donde los trastornos son más severos, teniendo presente, principalmente, trastornos del sueño, de la alimentación, dificultades counicativas, inflexibilidad mental y gran dificultad a la hora de aceptar cambios, como también presencia de conductas agresivas y/o auto agresivas.

En el caso de los trastornos en el sueño presentes en los TEA, suelen tener repercusiones importantes. Las madres manifiestan grandes inconvenientes en sus hijos a la hora de conciliar y mantener el sueño y esto, sin duda, afecta a las familias y su dinámica: "hasta el día de hoy tiene problemas para dormir y era también un desgaste físico nuestro, andábamos caminando toda la noche turnándonos con el papá, después que aparece Ana el conflicto pasó a ser además de la hermana" (participante 1) o "tiene trastorno del sueño, anoche se despertó a las cuatro de la mañana, después de despertó otro ratito, pero no descansa su cabeza y yo tampoco" (participante 5).

A su vez, la alimentación también es un desafío que requiere constante cuidado y atención en el día a día. Muchas veces, los niños con TEA presentan problemas en esta área, tanto con la ansiedad que genera ganas de comer constantemente, no saber cuándo parar o con la selectividad en los alimentos, rechazando algunos o no pudiendo comer prácticamente nada por problemas sensoriales: "tiene una ansiedad de comer que es horrible, terrible, se come todo, todo, vive abriendo la heladera" (participante 4); "tenés que estar siempre constantemente vigilándolos, todo el tiempo, donde vos te vayas un ratito ya te hicieron cualquier macana, porque ya agarraron para la cocina, ya agarraron la heladera, ya se llevaron cualquier cosa" (participante 3).

También hay otras conductas como se mencionó anteriormente que se vinculan al comportamiento, como las agresiones o autoagresiones. Muchas veces, la mayor dificultad e impotencia de parte de las familias parte de no entender cómo funcionan, 
dar respuesta a por qué se pega, por qué grita, qué es lo que quiere decir con eso.

En los TEA, las conductas disruptivas se vinculan en gran medida al déficit en el área de la comunicación, al no contar con el lenguaje como fuente de expresión o, en otros casos, de un nivel superior de funcionamiento sí lo tienen, pero no logran hacer uso de forma funcional y contextualizada. En ocasiones, las rabietas o "berrinches" (como lo llaman las madres) corresponden a manifestaciones de necesidades, de rechazo o de malestar físico o emocional. Hasta que no logran comprender esto, lo cual necesariamente necesita de la ayuda de profesionales y de familias que vivan situaciones similares, las madres se pueden ver desbordados por la situación:

(...) eso creo que es lo más desesperante como padre, el no poder entender a tu hijo (...) lo más complicado es que no sabés qué están pensando, qué es lo que les pasa, qué es lo que quieren, qué es lo que no quieren, se te retoban, se te tiran al piso, cuando no tienen lenguaje, ¿no? ¿ta? y ta, o cuando aprenden a expresarse de otra forma, bueno ta ,ahí es más fácil, pero igual los que tienen lenguaje, igual, les pasa estas cosas que le dan como ataques frente a algo que vos decís, pero qué pasó, qué fue lo que cambiơ" (participante 2).

En la medida en que las madres logran darle sentido a estas conductas, nace la posibilidad de mejorar el entorno y de adaptar las respuestas para que vayan disminuyendo. Las madres de hijos más grandes dan cuenta de la adaptación que se va generando, donde las dificultades siguen presentes, pero van adquiriendo más herramientas a la hora de enfrentarse a ellas.

Un aspecto importante que se vincula con la situación es el proceso de afrontamiento que esté transitando. Visualizar el hecho de que hay cosas que no va a poder lograr genera mucha angustia: "hay cosas que duelen, porque hay cosas que los ves que no se integra, ellos están solos y no se integran” (participante 5).

El grupo de madres dio cuenta de cómo este proceso va transformándose con el tiempo, las madres de hijos más pequeños manifestaron un mayor grado de angustia frente a las diferencias que presentan con niños sin TEA y las de los adultos en su discurso expresan explícitamente cómo a través del tiempo se va asimilando la situación de su hijo: "uno se va adaptando a las circunstancias, y por supuesto que hay factores que favorecen ese proceso. Si bien hay cosas que no desaparecen nunca, uno aprende a cómo manejarlas" (participante 2).

\section{Apoyos}

Para que este proceso de adaptación sea más saludable, las madres más grandes y con mayor experiencia dieron cuenta de lo fundamental de encontrar un espacio terapéutico para ir sobrellevando la situación y tener instancias para hablar con alguien de lo que les pasa y sienten, ya que la energía que requiere el cuidado más el proceso por el que uno va transitando a partir de tener un hijo con TEA, necesita de una gran fortaleza: "uno tiene que estar fortalecido para llevar adelante todo eso (...) 
tarde o temprano explotás, entonces cuando uno tiene la oportunidad de tratarlo, de trabajarlo eso con alguien, ta, que te van orientando, es mejor" (participante 1). Y concluye su experiencia:

Nunca hice terapia yo hasta hace dos años que tuve un pre infarto y ahí como que me mandaron, y ta y me di cuenta lo bueno que era y que bien me hubiera hecho haberla tenido antes por eso yo sugiero que cuando tengan la posibilidad recurran porque es un espacio en el que uno, este, puede largar todo sin lastimar a nadie, sin, y en realidad me doy cuenta que sí, que me hubiera hecho falta antes.

A su vez, tener contacto con familias que vivencian situaciones similares ayuda a que el camino sea transitado de otra forma, más acompañado y con otra contención que a veces no logran encontrar donde no tienen información de la situación: "uno siente que es liberador, a mí me pasaba al principio que nos juntábamos los pocos padres que éramos, y veíamos que no era el único que le pasaba lo mismo" (participante 3).

Además, se plantea como necesidad, por un lado, el espacio que se vincula a la contención emocional, a poder hablar libremente, teniendo el apoyo de un profesional y, por otro, una red de padres que, de acuerdo a las necesidades que vayan surgiendo, busquen alternativas grupalmente para hacerles frente. Las madres se sienten cómodas hablando y vinculándose con otras madres en su misma situación y se sienten comprendidas. Esta red representa un intento de generar los apoyos entre ellas mismas teniendo en cuenta que hoy no los están recibiendo.

A su vez, la presencia del asistente personal puede generar un apoyo para las madres, a la hora de realizar actividades con sus hijos y hasta generar evoluciones en la persona con discapacidad cuando encuentran alguien adecuado para el rol:

Me daba pánico salir con Amanda, ahora está con una cuidadora de BPS (se refiere al asistente personal otorgado por el Sistema Nacional Integrado de Cuidados) y me dice "tenemos que ir al centro a mirar vidrieras", y bueno me animo un poco con ella porque se entiende muy bien con Amanda, y le explica mucho, y bueno fuimos a tomar un helado (participante 6).

\section{Conclusiones}

Con respecto al bienestar emocional, las principales necesidades presentes tienen que ver con el tiempo libre; $y$ los principales generadores de estrés están relacionados con los problemas de conducta, la severidad del trastorno, el proceso de afrontamiento por el que esté pasando la madre y los trastornos del sueño y alimentación que pueden estar asociados al trastorno. Es relevante destacar que, el lugar del bienestar emocional como dimensión de la calidad de vida, se encuentra en directa relación con el lugar que tienen los apoyos relacionados con la persona con discapacidad. En la medida en que los apoyos están, se podría suponer que más necesidades de estas 
madres se ven satisfechas y más disminuye el nivel de estrés y de malestar. Sin duda, las madres representan en quien efectivamente recae el cuidado, lo cual pudieron reflejar en sus discursos y en sus experiencias y las consecuencias que genera que el cuidado de una persona dependiente recaiga únicamente en la mujer.

Se puede observar que la severidad del trastorno se relaciona con el estado emocional en forma importante. Los trastornos del sueño y de la alimentación, representan desafíos cotidianos que afectan la dinámica familiar y generan un gran agotamiento en las madres que son las que hacen las modificaciones más importantes en su rutina.

Tal como se ha señalado, el presente estudio es la etapa preliminar de un estudio mayor. Por lo tanto, si bien la limitación principal es lo acotado de la muestra, lo que no permite generalizar los resultados, la información obtenida parece estar en consonancia con los resultados establecidos por investigaciones internacionales citadas previamente en los antecedentes de este trabajo. Es decir, la severidad del TEA y la presencia de los trastornos del sueño son los elementos más importantes a la hora de establecer determinantes en alteraciones en el bienestar emocional en las madres. Además, también se pudo establecer que la información con la que las madres cuentan acerca del funcionamiento de su hijo y las características del trastorno tienen un papel fundamental. Tener información o formación en el tema genera seguridad, pudiendo entender a su hijo y, por lo tanto, estableciendo estrategias exitosas en la cotidianeidad acordes a sus necesidades y sus características.

A su vez, si este proceso de formación se ve acompañado por una contención emocional que tenga en cuenta las etapas de afrontamiento por procesos de identificación, teniendo a otras madres con quienes hablar que vivan situaciones similares, el proceso de tener un hijo con autismo no tendrá el mismo impacto. Por lo tanto, la formación de la familia y la intervención terapéutica, o los apoyos profesionales, son elementos que deben tomarse en cuenta a la hora de pensar estrategias para mejorar la calidad de vida familiar, ya que representa el mayor apoyo para la persona con discapacidad.

Finalmente, se plantea como reflexión general la importancia de tomar en cuenta el conocimiento de las madres acerca de las necesidades de sus hijos, su opinión en cuanto a la necesidad de apoyo, frente al conocimiento profesional que en ocasiones, parece no dar respuesta satisfactoria al respecto. Por otra parte, subrayar la importancia que le dan las madres a las agrupaciones de familiares como forma central para afrontar situaciones individuales y del colectivo.

\section{Referencias}

Aubeeluck, A. V., Buchanan, H. y Stupple, E. J. N. (2012). 'All the burden on all the carers': exploring quality of life with family caregivers of Huntington's disease patients. Quality of Life Research, 21(8), 1425-1435. HTTPS://DOI. ORG/10.1007/S11136-011-0062-X
Bagnato, M. J. (2009). Trabajadores con discapacidad intelectual. Abordaje de sus habilidades laborales. Montevideo: CSIC. 
Bagnato, M. J., Da Cunha, H. y Falero, B. (2011). Personas en situación de dependencia por discapacidad. Documento de trabajo para el debate. Montevideo: Grupo de Trabajo de Sistema Nacional del Cuidados / MIDES.

Beck, A., Hastings, R. P., Daley, D. y Stevenson, J. (2004). Pro-social behaviour and behaviour problems independently predict maternal stress. Journal of Intellectual \& Developmental Disability, 29(4), 339-349. $\quad$ HTTPS://DOI. ORG/10.1080/13668250400014509

BPS. (2015). Informe sobre Encuesta Longitudinal de Protección Social (Ronda 2012-2013).

Brasfield, C. (2008). An exploration of the stressors coping resources, and resiliency of rural mothers of children with special needs. US, ProQuest Information \& Learning, 69, 665.

Cabanyes Truffino, J. y García Villamisar, D. (2004). Identificación y diagnóstico precoz de los trastornos del espectro autista. Revista de Neurología, 39(1), 81-90. HTTPs://DOI. ORG/10.33588/RN.3901.2004098

Canal Bedia, R., García Primo, P., Touriño Aguilera E., Santos Borgujo, J., Martín Cilleros, M. V., Ferrari, M. J., ... Posada de la Paz, M. (2006). La detección precoz del autismo. Psychosocial Intervention, 1(15), 29-45.

Gómez, M. y Sabeh, E. (2001). Calidad de vida Evolución del concepto y su influencia en la investigación y la práctica. Salamanca: Instituto Universitario de Integración en la Comunidad, Facultad de Psicología, Universidad de Salamanca.

Gómez, V. (2008). El debate en torno a la regulación de la igualdad de género en la familia. Política $y$ Sociedad, 45(2), 13-28.

INE. (2011). Censo Nacional 2011. Informe final. Montevideo: INE.

Kobe, F. H. y Hammer, D. (1994). Parenting stress and depression in children with mental retardation and developmental disabilities. Research in Developmental Disabilities, 15(3), 209-221.

Lessenberry, B. M. y Rehfeldt, R. A. (2004). Evaluating stress levels of parents of children with disabilities. Exceptional Children, 70(2), 231-244. HTTPS://DOI.ORG/10.1177/001440290407000207

Marradi, A. (2007). Metodología de las ciencias sociales. Buenos Aires: Emecé.
Martin, S. y Baker, D. (2001). Families and children with severe disabilities: Daily lives, systems, and concerns. Presentado en American Association of Behavioral and Social Sciences Meeting, Las Vegas.

Martínez, M. y Bilbao León, M. (2008). Acercamiento a la realidad de las familias de personas con autismo. Psychosocial Intervention, 17(2), 215230.

Merino, M., Martínez, M., Cuesta, J., García, I. y Pérez de la Varga, L. (2012). Estrés y familias de personas con autismo. España: Federación Autismo Castilla y León.

Peralta López, F. y Arellano Torres, A. (2010). Familia y discapacidad. Una perspectiva teóricoaplicada del Enfoque Centrado en la Familia para promover la autodeterminación. Electronic Journal of Research in Educational Psychology, 8(3), 1339-1362.

Pozo, P. (2010). Adaptación psicológica en madres $y$ padres de personas con trastornos del espectro autista: Un estudio multidimensional (Tesis doctoral inédita). Universidad Nacional de Educación a Distancia, Madrid. Recuperado a partir de HTTP://E-SPACIO.UNED.ES/FEZ/ ESERV.PHP? PID = TESISUNED:PSICOLOGIAMPPOZO\&DSID=DOCUMENTO.PDF

Rodríguez, J. M. (2011). Métodos de investigación cualitativa. Revista de Investigación Silogismo, 8, 1-33.

Schalock, R.y Verdugo Alonso, M. (2007). El concepto de calidad de vida en los servicios y apoyos para personas con discapacidad intelectual. Siglo Cero, 38(4), 21-36.

Turnbull, A. (2003). La calidad de vida de la familia como resultado de los servicios: el nuevo paradigma. Siglo Cero, 34(3), 59-73.

Verdugo Alonso, M. (2002). Evaluación de la percepción de calidad de vida en la infancia. Psicothema, 14(1), 86-91.

Verdugo Alonso, M., Rodríguez Aguiella, A. y Sainz Modino, F. (2012). Escala de calidad de vida familiar. Manual de aplicación. Salamanca: INICO. 